\title{
WYD-like skew information measures
}

\author{
Frank Hansen
}

July 182012

\begin{abstract}
Following recent advances in the theory of operator monotone functions we introduce new classes of WYD-like skew information measures.
\end{abstract}

\section{Introduction}

Let $h$ be a positive operator monotone function defined in the positive halfaxis. We suppose $h$ is normalized such that $h(1)=1$, and that $h$ is neither constant nor the identity function.

Kawasaki and Nagisa [11] recently proved that the functions

$$
t \rightarrow \frac{(t-a)(t-b)}{(h(t)-h(a))\left(h^{\sharp}(t)-h^{\sharp}(b)\right)} \quad t>0
$$

are operator monotone for $a, b>0$, where $h^{\sharp}$ is the operator monotone function defined by setting

$$
h^{\sharp}(t)=\frac{t}{h(t)} \quad t>0 .
$$

This is an interesting observation since by setting $a=b=1$, choosing $h(t)=$ $t^{p}$ for $0<p<1$, and multiplying with a positive constant we obtain the operator monotone functions

$$
t \rightarrow p(1-p) \frac{(t-1)^{2}}{\left(t^{p}-1\right)\left(t^{1-p}-1\right)} \quad t>0 .
$$


These functions were first studied by Hasegawa and Petz [9] in the context of monotone metrics on the state space of a quantum mechanical system. Monotone metrics are generated by positive normalized operator monotone functions $f$ defined in the positive half-axis and satisfying the functional equation $f(t)=t f\left(t^{-1}\right)=f^{*}(t)$ for $t>0$. We refer to [7] for a modern account of the theory of operator monotone functions.

To each monotone metric there is an associated metric adjusted skew information which is a measure of quantum information under the presence of a conservation law. The metric adjusted skew informations associated with the monotone metrics generated by the functions in (1) are the much celebrated Wigner-Yanase-Dyson skew informations defined by

$$
I_{\rho}(p, A)=-\frac{1}{2} \operatorname{Tr}\left[\rho^{p}, A\right]\left[\rho^{1-p}, A\right] \quad 0<p<1,
$$

where $\rho$ is a state and $A$ is a conserved quantity.

The theorem of Kawasaki and Nagisa thus points to the existence of additional families of WYD-like skew informations that may be more computationally tractable than general metric adjusted skew informations.

The proof presented by Kawasaki and Nagisa is rather long and depends heavily on the theory of analytic functions. Following an idea in [2] we shall present a short proof that also entails a generalization of the theorem.

Theorem 1.1. Let $h$ be a normalized positive function defined in the positive half-axis, and suppose $h$ is neither constant nor the identity function. Consider the mathematical rule

$$
f(t)=\frac{(t-1)^{2}}{(h(t)-1)\left(h^{\sharp}(t)-1\right)} \quad t>0
$$

which may not define $f$ everywhere in the positive half-axis.

(i) If $h$ is operator monotone then the rule defines $f$ as a positive operator monotone function in the positive half-axis.

(ii) If $h$ and $h^{\#}$ are operator convex then the rule defines $f$ as an operator monotone decreasing function in the positive half-axis.

Proof. We know under both set of assumptions that $h$ and $h^{\#}$ are continuously differentiable functions, and by differentiation of $f^{\#}$ we readily obtain 
$h^{\prime}(1)+\left(h^{\#}\right)^{\prime}(1)=1$. For $t \neq 1$ the denominator in (2) may be written

$$
\begin{aligned}
(h(t)-1)\left(h^{\sharp}(t)-1\right) & =t-h(t)-h^{\#}(t)+1 \\
& =-\left(h(t)-1+h^{\#}(t)-1-(t-1)\right),
\end{aligned}
$$

and therefore

$$
\frac{(h(t)-1)\left(h^{\sharp}(t)-1\right)}{t-1}=-\left(\frac{h(t)-1}{t-1}+\frac{h^{\#}(t)-1}{t-1}-1\right) .
$$

We may thus write $f$ on the form

$$
f(t)=-\frac{t-1}{g(t)-1}
$$

where the function

$$
g(t)= \begin{cases}\frac{h(t)-1}{t-1}+\frac{h^{\#}(t)-1}{t-1} & t>0, t \neq 1 \\ 1 & t=1 .\end{cases}
$$

is continuous also in $t=1$.

If $h$ is operator monotone then so is $h^{\#}$, see for example [7, Corollary 4.2]. We then realize that both $h$ and $h^{\#}$ are operator concave in the positive halfaxis, see for example [7, Corollary 2.2]. By Bendat and Sherman's theorem [1] it follows that the positive function $g$ is operator monotone decreasing thus operator convex. By once more invoking Bendat and Sherman's theorem we obtain that $-f(t)^{-1}$ and hence $f(t)$ is operator monotone.

If $h$ and $h^{\#}$ are assumed operator convex then $g$ is operator monotone in the positive half-axis by Bendat and Sherman's theorem. As above it follows that $g$ is operator concave. By once more invoking Bendat and Sherman's theorem we obtain that $f(t)$ is well-defined and operator monotone decreasing in the positive half-axis. QED

If the function $h$ in the above theorem is operator monotone then both $0<h^{\prime}(1)<1$ and $0<\left(h^{\#}\right)^{\prime}(1)<1$, see for example [7, Corollary 4.7]. In addition $f(0)=\lim _{t \rightarrow 0} f(t)>0$.

Symmetry of a monotone metric 13 is expressed through the functional equation $f(t)=t f\left(t^{-1}\right)=f^{*}(t)$ for the generating operator monotone function. We also say that a function $f$ defined in the positive half-axis is symmetric if $f^{*}=f$. It is important to know when functions written on the form (2) are symmetric. The following lemma is a small exercise left to the reader. 
Lemma 1.2. Functions $f$ written on the form (2) are symmetric $\left(f=f^{*}\right)$ if either $h=h^{*}$ or $h=\tilde{h}$, where $\tilde{h}(t)=h\left(t^{-1}\right)^{-1}$ for $t>0$.

The functions $h(t)=t^{p}$ appearing in the WYD-metrics satisfy $h=\tilde{h}$.

The positive operator monotone functions defined in the positive half-axis satisfying $h=h^{*}$ are characterized in [4, Theorem 1], cf. also [5, 2, 6].

The positive operator monotone functions defined in the positive half-axis satisfying $h=\tilde{h}$ are characterized in [3, Theorem 1.1].

\section{WYD-like skew information measures}

Let $c$ be the Morozova-Cencov function of a regular monotone metric. It is given on the form

$$
c(x, y)=\frac{1}{y f\left(x y^{-1}\right)} \quad x, y>0,
$$

where $f$ is a positive normalized operator monotone function defined in the positive half-axis such that $f^{*}=f$ and $f(0)>0$.

The corresponding metric adjusted skew information is defined by

$$
I_{\rho}^{c}(A)=\frac{m(c)}{2} K_{\rho}^{c}(i[\rho, A], i[\rho, A]),
$$

where $m(c)=f(0)$ is the metric constant, and $K_{\rho}^{c}$ is the monotone metric generated by $f$, cf. [13, 9, 5]. It may be extended [5, Theorem 3.8] to positive semi-definite states $\rho$. The metric adjusted skew information may be written on the convenient form

$$
I_{\rho}^{c}(A)=\frac{m(c)}{2} \operatorname{Tr} A \hat{c}\left(L_{\rho}, R_{\rho}\right) A,
$$

where

$$
\hat{c}(x, y)=(x-y)^{2} c(x, y) \quad x, y>0,
$$

and $L_{\rho}$ and $R_{\rho}$ denote left and write multiplication with $\rho$.

It is because of this expression that functions written on the form (2) lead to simplified formulas. Indeed, after normalization of $f$, the corresponding function in (5) takes the form

$$
\hat{c}(x, y)=\frac{1}{h^{\prime}(1)\left(1-h^{\prime}(1)\right)}\left(x+y-y h\left(x y^{-1}\right)-y h^{\#}\left(x y^{-1}\right)\right)
$$


expressed additively in terms of the perspectives of $h$ and $h^{\#}$. If we insert these functions in (4) we obtain:

Theorem 2.1. Let $f$ be a symmetric function given on the form

$$
f(t)=h^{\prime}(1)\left(1-h^{\prime}(1)\right) \frac{(t-1)^{2}}{(h(t)-1)\left(h^{\sharp}(t)-1\right)} \quad t>0,
$$

where $h$ is a normalized operator monotone function. The corresponding metric adjusted skew information may be written

$$
I_{\rho}^{c}(A)=\frac{1}{2(1-h(0))}\left(2 \operatorname{Tr} \rho A^{2}-\operatorname{Tr}\left(A h\left(\Delta_{\rho}\right) A \rho+A h^{\#}\left(\Delta_{\rho}\right) A \rho\right)\right),
$$

where $\Delta_{\rho}$ is the modular operator given by

$$
\Delta_{\rho} A=\rho A \rho^{-1} .
$$

Example 2.2. If we chose $h(t)=t^{p}$ for $0<p<1$ then the expression in (6) reduces to

$$
\begin{aligned}
I_{\rho}^{c}(A) & =\frac{1}{2}\left(2 \operatorname{Tr} \rho A^{2}-\operatorname{Tr}\left(A\left(\Delta_{\rho}\right)^{p} A \rho+A\left(\Delta_{\rho}\right)^{-p} \rho A\right)\right) \\
& =\frac{1}{2}\left(2 \operatorname{Tr} \rho A^{2}-\operatorname{Tr}\left(A \rho^{p} A \rho^{1-p}+A \rho^{1-p} A \rho^{p}\right)\right) \\
& =-\frac{1}{2} \operatorname{Tr}\left[\rho^{p}, A\right] \cdot\left[\rho^{1-p}, A\right]
\end{aligned}
$$

and we recover, as expected, the Wigner-Yanase-Dyson skew information measures [14, 12].

\subsection{Examples of WYD-like skew information measures}

The functions,

$$
f_{\alpha}(t)=t^{\alpha}\left(\frac{1+t}{2}\right)^{1-2 \alpha} \quad t>0
$$

studied in [4] provide, for $0 \leq \alpha \leq 1$, a monotonous bridge between the largest and the smallest monotone metrics. They satisfies $f_{\alpha}^{*}=f_{\alpha}$ and $f_{\alpha}^{\#}=f_{1-\alpha}$ for $0 \leq \alpha \leq 1$. We therefore obtain 
Theorem 2.3. The formula

$I_{\rho}^{\alpha}(A)=\operatorname{Tr} \rho A^{2}-\frac{1}{2} \operatorname{Tr}\left[A\left(\frac{1+\Delta_{\rho}}{2}\right)^{1-2 \alpha} \rho^{\alpha} A \rho^{1-\alpha}+A\left(\frac{1+\Delta_{\rho}}{2}\right)^{-1+2 \alpha} \rho^{1-\alpha} A \rho^{\alpha}\right]$

defines for $0 \leq \alpha \leq 1$ a metric adjusted skew information.

The skew information measures defined in the preceding theorem share all of the general properties of the Wigner-Yanase-Dyson skew information measures, cf. [5] and [2, Theorem 1.2]. In particular, they are non-negative quantities bounded by the variance $\operatorname{Var}_{\rho}(\mathrm{A})$, and they are convex functions in the state variable $\rho$.

Notice that $I_{\rho}^{1 / 2}(A)$ is the Wigner-Yanase skew information.

The simplest operator monotone functions $f$ satisfying $f=\tilde{f}$ are given by $f(t)=t^{p}$ for $0 \leq p \leq 1$. It is rather hard to write down any other example although they exist in abundance. The function

$$
f(t)=\left(\frac{t}{1+t}\right)^{1+t}(1+t)^{1+\frac{1}{t}} \quad t>0
$$

is an example of an operator monotone function satisfying the functional equation $f(t)=\tilde{f}(t)=f\left(t^{-1}\right)^{-1}$, cf. [3]. Therefore, also this function generates, through Theorem 1.1 and Theorem 2.1, a WYD-like skew information measure.

\subsection{Unbounded measures of skew information}

It is the condition $f(0)>0$ for the operator monotone function generating the underlying monotone metric that makes the associated metric adjusted skew information bounded. A monotone metric with this property $(f(0)>0)$ is called regular.

The metric adjusted skew information vanishes if the underlying metric is not regular. It was therefore proposed in [2] to simply remove the factor $f(0) / 2$ and define the so-called unbounded metric adjusted skew information

$$
I_{\rho}^{c}(A)=K_{\rho}^{c}(i[\rho, A], i[\rho, A]),
$$

when the metric is not regular. This may happen when the generating function $h$ in Theorem 1.1 is operator convex.

An unbounded metric adjusted skew information shares all the general properties of metric adjusted skew informations including convexity in the 
state variable. The only exception is boundedness. It can therefore not be extended from the state manifold to the state space.

If we choose $h(t)=t^{p}$ for $-1<p<0$ or $1<p<2$ then both $h$ and $h^{\#}$ are operator convex and $h=\tilde{h}$. The associated function constructed in (2) takes after normalization the form

$$
f(t)=p(1-p) \frac{(t-1)^{2}}{\left(t^{p}-1\right)\left(t^{1-p}-1\right)} \quad t>0 .
$$

Since the normalization changes the sign we obtain that $f$ is operator monotone, and since $f(0)=0$ it generates the unbounded metric adjusted skew information

$$
I_{\rho}^{c}(A)=\frac{-1}{p(1-p)} \operatorname{Tr}\left[\rho^{p}, A\right] \cdot\left[\rho^{1-p}, A\right]
$$

These unbounded extensions of the WYD-skew information measures were already discussed by Hasegawa [8, Theorem 3], but not in the context of monotone metrics. Later they were studied by Jenčová and Ruskai [10], cf. also [2].

\section{References}

[1] J. Bendat and S. Sherman. Monotone and convex operator functions. Trans. Amer. Math. Soc., 79:58-71, 1955.

[2] L. Cai and F. Hansen. Metric-adjusted skew information: Convexity and restricted forms of superadditivity. Lett Math Phys, 93:1-13, 2010.

[3] F. Hansen. Selfadjoint means and operator monotone functions. Math. Ann., 256:29-35, 1981.

[4] F. Hansen. Characterizations of symmetric monotone metrics on the state space of quantum systems. Quantum Information and Computation, 6:597605, 2006.

[5] F. Hansen. Metric adjusted skew information. Proc. Natl. Acad. Sci. USA, 105:9909-9916, 2008.

[6] F. Hansen. Convexity of quantum $\chi^{2}$-divergence. Proc. Natl. Acad. Sci. USA, 108:10078-10080, 2011. 
[7] F. Hansen. The fast track to löwner's theorem. arXiv:1112.0098, 2011.

[8] H. Hasegawa. $\alpha$-Divergence of the non-commutative information geometry. Reports on Mathematical Physics, 33:87-93, 1993.

[9] H. Hasegawa and D. Petz. On the Rimannian metric of $\alpha$-entropies of density matrices. Lett. Math. Phys., 38:221-225, 1996.

[10] A. Jenčová and M.B. Ruskai. A unified treatment of convexity of relative entropy and related trace functions, with conditions for equality. Rev. Math. Phys., 22(9):1099-1121, 2010.

[11] M. Kawasaki and Nagisa M. Transforms on operator monotone functions. arXiv:1206.5452v1, 2012.

[12] E. Lieb. Convex trace functions and the Wigner-Yanase-Dyson conjecture. Advances in Math., 11:267-288, 1973.

[13] D. Petz. Monotone metrics on matrix spaces. Linear Algebra Appl., 244:81-96, 1996.

[14] E.P. Wigner and M.M. Yanase. Information contents of distributions. Proc. Natl. Acad. Sci. U.S.A., 49:910-918, 1963. 